PROCEEDINGS OF THE

AMERICAN MATHEMATICAL SOCIETY

Volume 126, Number 10, October 1998, Pages 3089-3096

S 0002-9939(98)04390-1

\title{
WEAK CONVERGENCES OF PROBABILITY MEASURES: A UNIFORM PRINCIPLE
}

\author{
JEAN B. LASSERRE
}

(Communicated by Stanley Sawyer)

\begin{abstract}
We consider a set $\prod$ of probability measures on a locally compact separable metric space. It is shown that a necessary and sufficient condition for (relative) sequential compactness of $\prod$ in various weak topologies (among which the vague, weak and setwise topologies) has the same simple form; i.e. a uniform principle has to hold in $\prod$. We also extend this uniform principle to some Köthe function spaces.
\end{abstract}

\section{INTRODUCTION}

Conditions for convergence of sequences of measures, in particular, the weak*, weak and setwise convergences of probability measures, are of primary interest in many areas, Probability and Control Theory being two of them.

For instance, a large class of optimal control problems (deterministic or stochastic, in discrete or continuous time) can be transformed into optimization problems over sets of (probability) measures (see e.g. [2], [4], [7], [8], [9], [11], [12]).

In this paper we consider a set $\prod$ of probability measures on a locally compact separable metric space. To each of the standard three weak convergences, namely the vague, weak and setwise convergence, corresponds a necessary and sufficient condition for relative sequential compactness of $\prod$. Those conditions are the uniform boundedness for the vague convergence, the tightness for the weak convergence and the uniform countable additivity for the setwise convergence (see e.g. [5]).

For the case of a compact space, Dieudonné [3] has considered other weak convergences of measures, using the space of Riemann integrable functions, and the space of bounded semi-continuous functions. Again, a weak* converging sequence also converges for the corresponding (stronger) weak topologies if and only if some uniform property holds.

In this paper, we show that those uniform properties can in fact be expressed in the same simple form that we call the uniform principle. The same uniform principle is also extended to weak convergences of sequences in some Köthe function spaces.

Received by the editors November 25, 1996 and, in revised form, March 10, 1997.

1991 Mathematics Subject Classification. Primary 60B05, 60B10, 28A33.

Key words and phrases. Sequences of measures, weak convergences of measures, Banach lattices, Köthe function spaces.

(C) 1998 American Mathematical Society 


\section{WEAK CONVERGENCES OF PROBABILITY MEASURES}

2.1. Notation and definitions. Let $X$ be a locally compact separable metric space and $\mathcal{B}$ its usual Borel $\sigma$-algebra. Let $X_{0}:=C_{0}(X)$ be the Banach space of continuous functions on $X$ that vanish at infinity, $X_{1}:=C_{b}(X)$ the Banach spaces of bounded continuous functions on $X$ and $X_{2}:=B(X)$ the Banach space of bounded measurable functions on $(X, \mathcal{B})$, all equipped with the sup-norm. Of course

$$
X_{0} \subset X_{1} \subset X_{2}
$$

$X_{0}^{*}$ is isometrically isomorphic to $r c a(X)$, the Banach space of finite signed Borel measures on $(X, \mathcal{B})$, whereas $X_{1}^{*}$ is isometrically isomorphic to $r b a(X)$, the Banach space of finitely additive finite signed regular measures on $(X, \mathcal{B})$ and $X_{2}^{*}$ is isometrically isomorphic to $b a(X)$, the Banach space of finitely additive finite signed measures on $(X, \mathcal{B})$ (see e.g. $[6])$.

With the usual partial ordering $\leq$ on $X_{i}$, both $X_{i}$ and $X_{i}^{*}$ are Banach lattices for $i=0,1,2$.

In $X_{0}^{*}$, consider the three weak topologies $\sigma\left(X_{0}^{*}, X_{i}\right), i=0,1,2$, with respective convergence denoted by " $\rightarrow$ " convergence is the usual vague convergence, whereas the w1-convergence is the weak convergence and the $w 2$-convergence is the setwise convergence. The weak convergence is sometimes denoted by $\Rightarrow$.

A set $K \subset r c a(X)$ is $w i$-sequentially compact (in short $w i$-s.c.) if every sequence has a converging subsequence with limit in $r c a(X)$ but not necessarily in $K$. It is well-known that for a set $\Pi \subset \operatorname{rca}(X)$ of probability measures:

$$
\prod \text { is } w 0 \text {-s.c. since } \sup _{\mu \in \prod}\|\mu\|=1<\infty,
$$

and the unit ball in $r c a(X)$ is weak* sequentially compact. In addition,

$$
\prod \text { is } w 1 \text {-s.c. } \Leftrightarrow \forall \epsilon>0, \exists \text { compact } K \subset \mathcal{B} \text { with } \inf _{\mu \in \prod} \mu(K) \geq 1-\epsilon \text {. }
$$

Finally,

$$
\prod \text { is } w 2 \text {-s.c. } \Leftrightarrow \text { the countable additivity is uniform in } \prod \text {. }
$$

Thus, in view of (2.2), (2.3) and (2.4), to get the $w i$-sequential compactness of $\prod$, a uniform property needs to hold in $\prod$, the uniform boundedness in $(2.2)$, tightness in (2.3) and the uniform countable additivity in (2.4).

Note also that the three weak topologies considered are nothing less than the weak $^{*}$ topology when $\prod$ is considered as a subset of $r c a(X), \operatorname{rba}(X)$ or $b a(X)$.

For the case of a compact metric space $X$, and given a set $\prod \subset \operatorname{rca}(X)$, following Dieudonné [3], we also consider two other spaces of functions on $X$, namely the space $X_{3}$ of Riemann integrable functions, i.e. the functions that are bounded and continuous, except at points in a set (depending on the considered function) of null measure for every $\mu \in \prod$ and the space $X_{4}$ of bounded semi-continuous functions on $X$.

Again, it has been shown in [3], that if a sequence $\mu_{n} \in \prod$ converges to some $\mu \in \operatorname{rca}(X)$ for the weak* topology, then

$$
\int f d \mu_{n} \rightarrow \int f d \mu \forall f \in X_{i} \text { with } i=3 \text { or } 4,
$$


if and only if

$$
\forall \epsilon>0, \forall K \text { compact (with } \mu_{n}(K)=0 \forall n \text { if } i=4 \text { ). }
$$

(2.5) $\exists O$ open neighborhood of $K$, s.t. $\mu_{n}(H) \leq \epsilon \forall n, \forall$ compact $H \subset O-K$.

Again, (2.5) is a uniform property of the sequence $\left\{\mu_{n}\right\}$.

2.2. Weak convergences: a uniform principle. We show that the uniform properties described in the above section can in fact be stated in the same simple compact form.

Theorem 2.1. Let $i \in\{0,1,2,3,4\}$ be fixed, in which case $X$ is assumed to be compact if $i=3$ or 4 . Let $\left\{\mu_{n}\right\}$ be sequence of probability measures in rca $(X)$ that converges to $\mu$ for the $\sigma\left(r c a(X), X_{0}\right)$ (or weak*) topology. Then,

$$
\int f d \mu_{n} \rightarrow \int f d \mu \forall f \in X_{i}
$$

if and only if

$$
\lim _{k \rightarrow \infty} \sup _{n} \int f_{k} d \mu_{n} \rightarrow 0 \quad \text { whenever } f_{k} \downarrow 0, f_{k} \in X_{i} .
$$

Proof. The only if part. Assume that (2.6) holds. The case $i=0$ is straightforward since

$$
\sup _{n} \int f_{k} d \mu_{n} \leq\left\|f_{k}\right\| \downarrow 0
$$

since $X_{0}=C_{0}(X)$ is an order-continuous Banach lattice.

Consider an arbitrary sequence $\left\{f_{k}\right\}$ in $X_{i}$ with $f_{k} \downarrow 0$. From the convergence of $\mu_{n}$ to $\mu$ we get:

$$
\forall \epsilon>0 \forall k \exists n(k, \epsilon) \text { with }\left|\int f_{k} d \mu_{n}-\int f_{k} d \mu\right| \leq \epsilon, \forall n \geq n(k, \epsilon) .
$$

As $\mu \in \operatorname{rca}(X)$, from $f_{k} \downarrow 0$

$$
\forall \epsilon>0 \exists k_{0} \int f_{k} d \mu \leq \epsilon \forall k \geq k_{0} .
$$

Fix $\epsilon>0$, arbitrary, and $k_{0}$ as in (2.9). From (2.8) and (2.9), $\exists n\left(k_{0}, \epsilon\right)$ such that

$$
\int f_{k} d \mu_{n} \leq 2 \epsilon \forall n \geq n\left(k_{0}, \epsilon\right) \text { and } \forall k \geq k_{0}
$$

(since $f_{k} \geq f_{k+1} \forall k$ ). Therefore:

$$
\sup _{n \geq n\left(k_{0}, \epsilon\right)} \int f_{k} d \mu_{n} \leq 2 \epsilon \forall k \geq k_{0} .
$$

Now, for every $n \leq n\left(k_{0}, \epsilon\right), \exists k(n)$ such that $\int f_{k} d \mu_{n} \leq 2 \epsilon \forall k \geq k(n)$ since $\mu_{n} \in$ $r c a(X)$ and $f_{k} \downarrow 0$.

Therefore, take

$$
k^{\prime}:=\max \left\{k_{0}, \max _{n=1, . . n\left(k_{0}, \epsilon\right)} k(n)\right\}
$$

to obtain

$$
\sup _{n} \int f_{k} d \mu_{n} \leq 2 \epsilon \forall k \geq k^{\prime}
$$


and thus, as $\epsilon$ was arbitrary,

$$
\lim _{k \rightarrow \infty} \sup _{n} \int f_{k} d \mu_{n}=0
$$

i.e. (2.7) holds.

The if part. The case $i=0$ is trivial.

(a) For the case $i=1$, i.e. $X_{i}:=C_{b}(X)$.

As $X$ is a locally compact and separable metric space, consider an increasing sequence of compact sets $K_{n} \uparrow X$ and open sets $O_{n}$ with the properties

$$
K_{n} \subset O_{n} \subset K_{n+1} \forall n .
$$

By Urysohn's Lemma one may construct $0 \leq f_{n} \leq 1 \in C_{b}(X)$, with

$$
f_{n}=1, x \in K_{n} ; f_{n}=0, x \in O_{n}^{c} n=0, \ldots
$$

so that $f_{n} \uparrow 1$. As $f_{n} \uparrow 1$, the sequence $\left\{g_{n}\right\}$ in $C_{b}(X)$ with $g_{n}:=1-f_{n} \downarrow 0$. Then,

$$
\int g_{i} d \mu_{n}=1-\int f_{i} d \mu_{n} \geq 1-\mu_{n}\left(K_{i+1}\right)=\mu_{n}\left(K_{i+1}^{c}\right)
$$

so that from $(2.7)$ we get:

$$
\forall \epsilon>0, \exists i_{0}, \sup _{n} \mu_{n}\left(K_{i}^{c}\right) \leq \epsilon \forall i \geq i_{0},
$$

i.e. the sequence $\left\{\mu_{n}\right\}$ is tight and therefore, by Prohorov's Theorem, is sequentially compact in the weak topology $\sigma\left(\operatorname{rca}(X), X_{1}\right)$. Hence, $\mu_{n_{k}} \Rightarrow \nu$ for some subsequence $\left\{\mu_{n_{k}}\right\}$ and some $\nu \in \operatorname{rca}(X)$.

But as $\mu_{n} \rightarrow \mu$ in the weak* topology, we necessarily have $\mu=\nu$, and as $\nu$ was an arbitrary weak limit point, all the weak limit points are equal to $\mu$ and $\mu_{n} \Rightarrow \mu$, i.e. (2.6) holds.

(b) The case $i=2$, i.e. $X_{2}=B(X)$. Take an arbitrary sequence of Borel sets $O_{k} \in \mathcal{B}$ with $O_{k} \downarrow \emptyset$ and define $f_{k}:=1_{O_{k}} \in B(X)$. Thus, $f_{k} \downarrow 0$. From (2.7)

$$
\lim _{k \rightarrow \infty} \sup _{n} \mu_{n}\left(O_{k}\right)=0,
$$

i.e., the countable additivity of $\mu_{n}$ is uniform in $\prod$ and thus, $\prod$ is $\sigma\left(r c a(X), r c a(X)^{*}\right)$ sequentially compact (see e.g. [6]). Hence, there is a subsequence $\mu_{n_{k}}$ that converges to some $\nu \in \operatorname{rca}(X)$. But therefore, $\nu=\mu$ for every weak limit point $\nu$, which implies that $\mu_{n} \rightarrow \mu$ in the $\sigma\left(r c a(X), r c a(X)^{*}\right)$ topology. Since the $\sigma\left(r c a(X), r c a(X)^{*}\right)$ topology is stronger than the $\sigma\left(r c a(X), X_{2}\right)$ topology, the proof is complete.

(c) For the case $i=3$ when $X$ is a compact metric space, let $K$ be a compact set in $X$ with $\mu_{n}(K)=0 \forall n$. Let $O_{j}$ be a decreasing sequence of open sets with $K \subset O_{j}, \overline{O_{j+1}} \subset O_{j} \forall j$, and $\lim _{j} \overline{O_{j}}=K$. Then, by Urysohn's lemma, construct $g_{j} \in X_{2}, 0 \leq g_{j} \leq 1$, as follows:

$$
g_{j}(x)=1 \forall x \in \overline{O_{j+1}}, g_{j}(x)=0 \forall x \in O_{j}^{c},
$$

and a function $f_{j}$ satisfying

$$
f_{j}(x)=g_{j}(x) \forall x \in K^{c}, f_{j}(x)=0 \forall x \in K .
$$

Obviously, $f_{j} \in X_{3}$ since $f_{j}$ is continuous except at points in $K$. In addition, $f_{j} \downarrow 0$ and $\int f_{j} d \mu_{n} \geq \mu_{n}(H)$ for every compact $H \subset O_{j+1}-K$. But, from (2.7), for an arbitrary $\epsilon>0, \mu_{n}(H) \leq \epsilon$ provided $j$ is large enough, i.e. the condition $C_{I I}$ in Prop. 4 [3], p. 29, i.e. (2.5) with $i=3$, is satisfied and thus $\int f d \mu_{n} \rightarrow \int f d \mu \forall f \in X_{3}$. 
(d) For the case $i=4$, the proof is the same as for (c). Indeed, consider any compact set $K \in \mathcal{B}$. The function $f_{j}$ defined in (2.10) is lower-semi-continuous and thus belongs to $X_{4}$. The condition $C_{I I I}$ in Prop.5 in [3] p. 32, i.e. (2.5) with $i=4$, is satisfied so that $\int f d \mu_{n} \rightarrow \int f d \mu \forall f \in X_{4}$.

We thus have:

Corollary 2.2. Let $\Pi \subset \operatorname{rca}(X)$ be a set of probability measures, and let $i \in$ $\{0,1,2,3,4\}$ be fixed. When $i=3$ or $i=4, X$ is assumed to be compact. Then the following two propositions are equivalent:

(a) $\prod$ is sequentially compact in the $\sigma\left(r c a(X), X_{i}\right)$ topology.

(b) $\lim _{k \rightarrow \infty} \sup _{\mu \in \prod} \int f_{k} d \mu=0$ whenever $f_{k} \downarrow 0$ with $f_{k} \in X_{i}$.

Proof. (b) $\Rightarrow$ (a). The case $i=0$ is trivial since the weak ${ }^{*}$ sequential compactness follows from the uniform norm-boundedness of $\prod$ and the fact that $X_{0}=C_{0}(X)$ is separable.

The cases $i=1,2,3,4$. Fix $i \in\{1,2,3,4\}$. $\prod$ is weak* sequentially compact. Therefore, every sequence $\left\{\mu_{n}\right\}$ in $\prod$ contains a subsequence $\mu_{n_{k}}$ that converges to $\mu \in \operatorname{rca}(X)$ for the $\sigma\left(r c a(X), X_{0}\right)$ topology. As (b) holds, from Theorem 2.1,

$$
\int f d \mu_{n_{k}} \rightarrow \int f d \mu \forall f \in X_{i}
$$

and the conclusion follows.

(a) $\Rightarrow$ (b). Assume that (b) does not hold. Then,

$$
\exists \epsilon>0, \forall k_{0} \exists k \geq k_{0}, \exists \mu_{k} \in \prod \text { with } \int f_{k} d \mu_{k}>\epsilon
$$

for some sequence $f_{k} \downarrow 0, f_{k} \in X_{i}$. Therefore, let $k_{0} \rightarrow \infty$. There is a sequence $\left\{\mu_{n}\right\}$ in $\prod$ with

$$
\int f_{n} d \mu_{n}>\epsilon \forall n
$$

However, as $\prod$ is sequentially compact, $\mu_{n_{k}} \rightarrow \mu$ for some $\mu \in \operatorname{rca}(X)$ and some subsequence $\mu_{n_{k}}$ in the $\sigma\left(r c a(X), X_{i}\right)$ topology.

But from Theorem 2.1 we then must have

$$
\lim _{k \rightarrow \infty} \sup _{m} \int f_{k} d \mu_{n_{m}}=0 .
$$

In particular, there is some $k_{0}$ such that

$$
\int f_{k} d \mu_{n_{m}} \leq \epsilon / 2 \forall m, \forall k \geq k_{0}
$$

a contradiction with (2.12).

Corollary 2.2(b) states a uniform principle for the weak sequential compactness of $\prod$ in the five weak topologies considered. The conditions $(2.2)-(2.4)$, and (2.5) are just specialized versions of this uniform principle.

Example. Consider $\prod$, the sequence of Dirac measures $\delta_{x_{i}}$ on $((0, \infty), \mathcal{B})$ with $x_{i} \rightarrow 0 \notin X$ ( $X$ is not complete). Then $\delta_{x_{i}} \rightarrow 0$ in the weak* topology since the functions in $C_{0}(X)$ must satisfy $f\left(x_{i}\right) \rightarrow 0$ whenever $x_{i} \rightarrow 0$. 
However $\delta_{x_{i}} \nRightarrow 0$ since $\delta_{x_{i}} \Rightarrow \delta_{0} \notin r c a(X)$. Let $0 \downarrow f_{k} \in C_{b}(X)$ with

$$
f_{k}(x):=1 \text { on }(0,1 / k], 2-k x \text { on }[1 / k, 2 / k], 0 \text { on }[2 / k, \infty), k=1, \ldots,
$$

$\sup _{n} \int f_{k} d \delta_{x_{n}}=1 \Rightarrow \prod$ is not $\sigma\left(r c a(X), C_{b}(X)\right)$-sequentially compact.

With the same example on now $X:=[0, \infty), \delta_{x_{i}} \Rightarrow \delta_{0}$ but $\delta_{x_{i}}$ does not converge setwise to $\delta_{0}$. Indeed, the same sequence $f_{k}$ with $f_{k}(0)=0$ is now in $B(X)$, but not in $C_{b}(X)$. Again, it satisfies $f_{k} \downarrow 0$ and $\sup _{n} \int f_{k} d \delta_{x_{n}}=1$.

We have seen that (b) in Corollary 2.2 with $i=1$ is equivalent to tightness of $\prod$, a simpler criterion to check since it amounts to checking (b) for just a single sequence $f_{n}:=1_{O_{n}}$ (with $O_{n}$ open and $\left.O_{n} \downarrow \emptyset\right)$. However, note that this single sequence $\left\{f_{n}\right\} \not \subset C_{b}(X)$.

An interesting question is "is there an equivalent criterion, but simpler than (b), for the case $i=2$ ?". A sufficient condition is e.g. that $\prod$ be order-bounded from above by a measure in $r c a(X)$. Also, can we extend this result to spaces other than $X_{k}, k=1, \ldots, 4$ ? The next section provides an answer to the latter question.

\section{WEAK CONVERGENCES IN KÖthe FUnCTION SPACES}

Let $\mathcal{X}$ and $\mathcal{Y}$ be Banach lattices with $\mathcal{X} \subset \mathcal{Y}$ and assume that $\mathcal{X}$ is separable and $\sigma$-complete (hence order-continuous).

It is also assumed that both $\mathcal{X}$ and $\mathcal{Y}$ are Köthe function spaces on the same (complete) measure space $(\Omega, \mathcal{B}, \mu)$.

For a Köthe function space $\mathcal{Y}$ with dual $\mathcal{Y}^{*}$, let $\mathcal{Y}^{\prime} \subset \mathcal{Y}^{*}$ be the elements that are integrals (cf. [10]). $\mathcal{Y}^{\prime}$ is an ideal of $\mathcal{Y}^{*}$, i.e. if $y^{*} \in \mathcal{Y}^{*}$, then $y^{*} \in \mathcal{Y}^{\prime}$ whenever $\left|y^{*}\right| \leq\left|z^{*}\right|$ for some $z^{*} \in \mathcal{Y}^{\prime}$. An element $y^{*} \in \mathcal{Y}^{\prime}$ if and only if (see [10])

$$
\lim _{k \rightarrow \infty}\left|y^{*}\right|\left(y_{k}\right) \rightarrow 0 \quad \text { whenever } y_{k} \downarrow 0, y_{k} \in \mathcal{Y} .
$$

As $\mathcal{X}$ is $\sigma$-order continuous, $\mathcal{X}^{*}=\mathcal{X}^{\prime}$, i.e. the elements of $\mathcal{X}^{*}$ are all integrals (see e.g. [10]) and therefore $\mathcal{X}^{*} \subset \mathcal{Y}^{\prime}$. We also assume that $\mathcal{X}^{*}=\mathcal{X}^{\prime}=\mathcal{Y}^{\prime}$.

We are interested in the following question. Given a nonnegative sequence $\left\{x_{n}^{*}\right\} \subset \mathcal{X}^{*}$ which converges to some $x^{*} \in \mathcal{X}$ for the weak* topology $\sigma\left(\mathcal{X}^{*}, \mathcal{X}\right)$, under what condition does this sequence also converge to $x^{*}$ for the stronger $\sigma\left(\mathcal{X}^{*}, \mathcal{Y}\right)$ topology?

Theorem 3.1. (a) Let $\left\{x_{n}^{*}\right\}$ be a nonnegative sequence in $\mathcal{X}^{*}$ that converges to some $x^{*} \in \mathcal{X}^{*}$ for the weak* topology. Then

$$
x_{n}^{*}(y) \rightarrow x^{*}(y) \forall y \in \mathcal{Y},
$$

(i.e. $x_{n}^{*} \rightarrow x^{*}$ for the $\sigma\left(\mathcal{X}^{*}, \mathcal{Y}\right)$ weak topology) if and only if

$$
\lim _{k \rightarrow \infty} \sup _{n} x_{n}^{*}\left(y_{k}\right) \downarrow 0 \quad \text { whenever } y_{k} \downarrow 0 \text { in } \mathcal{Y} .
$$

(b) Every nonnegative norm-bounded set $K \subset \mathcal{X}^{*}$ is $\sigma\left(\mathcal{X}^{*}, \mathcal{Y}\right)$-sequentially compact if and only if

$$
\lim _{k \rightarrow \infty} \sup _{x^{*} \in K} x^{*}\left(y_{k}\right) \downarrow 0 \text { whenever } y_{k} \downarrow 0 \text { in } \mathcal{Y} .
$$

Proof. (a) The if part. From the weak* convergence of $x_{n}^{*}$ to $x^{*}$, the sequence $\left\{x_{n}^{*}\right\}$ is norm-bounded. From the weak* compactness of the unit ball in $\mathcal{Y}^{*}$, there is also 
a subnet (cf. [1]) $\left\{x_{\alpha}^{*}, \alpha \in D\right\}$ of $\left\{x_{n}^{*}\right\}$ (for some directed set $D$ ) that converges to some $0 \leq y^{*} \in \mathcal{Y}^{*}$, i.e.

$$
x_{\alpha}^{*}(y) \rightarrow y^{*}(y) \forall y \in \mathcal{Y} .
$$

However, from (3.3), $x_{n}^{*}\left(y_{i}\right) \downarrow 0$ uniformly, whenever $y_{i} \downarrow 0$. Therefore, with $\epsilon>0$ fixed, arbitrary,

$$
\exists i_{0},\left|x_{n}^{*}\left(y_{i}\right)\right| \leq \epsilon \forall i \geq i_{0}, \forall n
$$

In addition, for any $\epsilon>0$ and $y_{i}$ fixed with $i \geq i_{0}$, from the convergence of $x_{\alpha}^{*}$ to $y^{*} \in \mathcal{Y}^{*}$, we get

$$
\left|x_{\alpha}^{*}\left(y_{i}\right)-y^{*}\left(y_{i}\right)\right| \leq \epsilon \forall \alpha \geq \alpha_{0} .
$$

so that $\left|y^{*}\left(y_{i}\right)\right| \leq 2 \epsilon \forall i \geq i_{0}$. As $\epsilon$ was arbitrary we conclude that $y^{*}\left(y_{i}\right) \downarrow 0$ whenever $y_{i} \downarrow 0$. From (3.1) $y^{*} \in \mathcal{X}^{*}$. But then, we must have $x^{*}=y^{*}$. As $y^{*}$ was an arbitrary weak limit point, all the weak limit points are in fact equal to $x^{*}$, i.e. $x_{n}^{*}$ converges to $x^{*}$ in the $\sigma\left(\mathcal{X}^{*}, \mathcal{Y}\right)$ weak topology, the desired result.

The only if part. Assume that $x_{n}^{*}(y) \rightarrow x^{*}(y) \forall y \in \mathcal{Y}$. Consider a sequence $y_{k} \downarrow 0$, $y_{k} \in \mathcal{Y}$. Then:

$$
\forall \epsilon>0 \forall k \exists n(k, \epsilon) \text { with }\left|x_{n}^{*}\left(y_{k}\right)-x^{*}\left(y_{k}\right)\right| \leq \epsilon \forall n \geq n(k, \epsilon) .
$$

As $x^{*} \in \mathcal{X}^{*}=\mathcal{Y}^{\prime}$ and $y_{k} \downarrow 0$

$$
\forall \epsilon>0 \exists k_{0} x^{*}\left(y_{k}\right) \leq \epsilon \forall k \geq k_{0} .
$$

Fix $\epsilon>0$, arbitrary, and $k_{0}$ as in (3.6). From (3.5) and (3.6), $\exists n\left(k_{0}, \epsilon\right)$ such that

$$
x_{n}^{*}\left(y_{k}\right) \leq 2 \epsilon \forall k \geq k_{0} \forall n \geq n\left(k_{0}, \epsilon\right)
$$

since $y_{k} \geq y_{k+1} \forall k$. Therefore,

$$
\sup _{n \geq n\left(k_{0}, \epsilon\right)} x_{n}^{*}\left(y_{k}\right) \leq 2 \epsilon \forall k \geq k_{0} .
$$

Now, for every $n \leq n\left(k_{0}, \epsilon\right), \exists k(n)$ such that $x_{n}^{*}\left(y_{k}\right) \leq 2 \epsilon \forall k \geq k(n)$ since $y_{k} \downarrow 0$ and $0 \leq x_{n}^{*} \in \mathcal{X}^{*}=\mathcal{Y}^{\prime}$. Therefore, with $k^{\prime}:=\max \left[k_{0}, \max _{n=1, . . n\left(k_{0}, \epsilon\right)} k(n)\right]$ we obtain

$$
\sup _{n} x_{n}^{*}\left(y_{k}\right) \leq 2 \epsilon \forall k \geq k^{\prime}
$$

As $\epsilon$ was arbitrary we conclude that

$$
\lim _{k \rightarrow \infty} \sup _{n} x_{n}^{*}\left(y_{k}\right) \rightarrow 0,
$$

the desired result.

(b) The if part. As $\mathcal{X}$ is separable, the unit ball of $\mathcal{X}^{*}$ is weak* sequentially compact, i.e. in the $\sigma\left(\mathcal{X}^{*}, \mathcal{X}\right)$ topology. Therefore, any sequence in $K$ has a converging subsequence in $\mathcal{X}^{*}$. From (a) this subsequence also converges in the $\sigma\left(\mathcal{X}^{*}, \mathcal{Y}\right)$ topology.

The only if part. Assume that (3.4) does not hold. Then,

$$
\exists \epsilon>0 \forall k_{0} \exists k \geq k_{0}, \exists x_{k}^{*} \in K \text { with } x_{k}^{*}\left(y_{k}\right) \geq \epsilon,
$$

for some sequence $y_{k} \downarrow 0, y_{k} \in \mathcal{Y}$. 
Let $k_{0} \rightarrow \infty$. There is a sequence $\left\{y_{n}, x_{n}^{*}\right\}$ with $x_{n}^{*}\left(y_{n}\right) \geq \epsilon \forall n$. However, as $K$ is $\sigma\left(\mathcal{X}^{*}, \mathcal{Y}\right)$-sequentially compact, $x_{n_{i}}^{*} \rightarrow x^{*} \in \mathcal{X}^{*}$ for some subsequence and some $x^{*} \in \mathcal{X}^{*}$. But from (a) we then must have

$$
\lim _{k \rightarrow \infty} \sup _{i} x_{n_{i}}^{*}\left(y_{k}\right)=0,
$$

a contradiction with (3.8).

Now, observe that with $X$ as in Section $2, \mathcal{Y}:=B(X)$ is an abstract $M$-space (with even a strong unit) so that $\mathcal{Y}^{*}$ is order-isometric to $L_{1}(\Omega, \mathcal{B}, \mu)$ for some measure space $(\Omega, \mathcal{B}, \mu)$. In turn, by the natural embedding of $\mathcal{Y}$ into $\mathcal{Y}^{* *}, \mathcal{Y}$ is order-isometric to a sublattice of $L_{\infty}(\Omega, \mathcal{B}, \mu)$, a Köthe function space (see e.g. [10]. The same argument holds with $\mathcal{Y}:=C_{b}(X)$ which is also an abstract $M$-space.

In addition, $\mathcal{X}:=C_{0}(X)$ is a sublattice of $\mathcal{Y}$ and thus order-isometric to a sublattice of $L_{\infty}(\Omega, \mathcal{B}, \mu)$. Being separable and $\sigma$-complete, it is order-continuous so that $\mathcal{X}^{\prime}=\mathcal{X}^{*}($ see $[10])$.

\section{REFERENCES}

1. R.B. Ash, Real Analysis and Probability, Academic Press, New York, 1972. MR 55:8280

2. E.J. Balder, On compactness of the space of policies in stochastic dynamic programming, Stoch. Proc. Appl. 32, pp. 141-150, 1989. MR 91b:90212

3. J. Dieudonné, Sur la convergence des suites de mesures de Radon, Anais Acad. Brasil. Ci. 23, pp. 21-38, 1951. MR 13:121a

4. A.G. Bhatt, V. Borkar, Occupation measures for controlled Markov processes: characterization and optimality, Ann. Prob. 24 (1996), 1531-1562. MR 97i:90105

5. J.L. Doob, Measure Theory, Springer-Verlag, New York, 1994. MR 95c:28001

6. N. Dunford, J.T. Schwartz, Linear Operators. Part I: General Theory, Interscience Publishers, Inc., New York, 1957. MR 90g:47001a

7. W.H. Fleming, D. Vermes, Convex duality approach to the optimal control of diffusions, SIAM J. Contr. Optim. 27, pp. 1136-1155, 1989. MR 90i:49016

8. O. Hernandez-Lerma, J.B. Lasserre, Discrete-Time Markov Control Processes: Basic Optimality Criteria, Springer-Verlag, New York, 1996. MR 96k:93001

9. M. Kurano, M. Kawai, Existence of optimal stationary policies in discounted decision processes, Comp. Math. Appl. 27, pp. 95-101, 1994. MR 95a:90197

10. J. Lindenstrauss, L. Tzafriri, Classical Banach spaces I and II, Springer-Verlag, Berlin, 1977. MR 81c:46001; MR 58:17766

11. E. Mascolo, L. Migliaccio, Relaxation methods in control theory, Appl. Math. Optim. 20, pp. 97-103, 1989. MR 90c: 49059

12. J.E. Rubio, The global control of nonlinear diffusion equations, SIAM J. Contr. Optim. 33, pp. 308-322, 1995. MR 90b:49009

13. K. Yosida, Functional Analysis, Springer-Verlag, New York, 1980. MR 82i:46002

LAAS-CNRS, 7 Av. du Colonel Roche, 31077 Toulouse Cédex, France

E-mail address: lasserre@laas.fr 